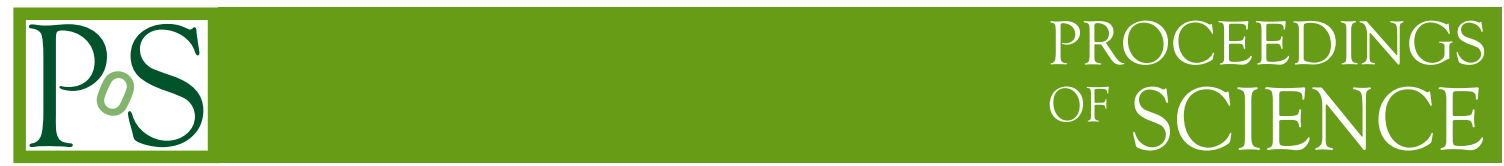

\title{
Generation of magnetic fields in cosmic string wakes
}

\author{
Sovan Sau ${ }^{a * *}$ and Soma Sanyal ${ }^{a}$ \\ ${ }^{a}$ University of Hyderabad, \\ Prof. C. R. Rao Road, Hyderabad, 500046, India \\ E-mail: sovan.sau@gmail.com, sossp.uoh@nic.in
}

\begin{abstract}
We describe a novel method of generating magnetic fields in cosmic string wakes from neutrino currents. We show that neutrino currents act as a cross-perturbation across the cosmic string wake. This cross perturbation along with the high Reynolds number generates a magnetic field in the wake of the cosmic string. The neutrino current is generated by the neutrinos rotating around the Abelian Higgs strings. As the string moves through the cosmic plasma, the velocity kick generated by the motion of the string will enhance the neutrino current in the wake region. The neutrino current density depends on its distance from the string and is oscillatory in nature. This leads to neutrino density gradients in the plasma. We have shown that these neutrino gradients give rise to electron gradients in the plasma, which in turn generate magnetic fields of the order of $10^{13}$ Gauss.
\end{abstract}

40th International Conference on High Energy physics - ICHEP2020

July 28 - August 6, 2020

Prague, Czech Republic (virtual meeting)

\footnotetext{
${ }^{*}$ Speaker
} 


\section{Introduction}

Abelian Higgs strings are linear topological defects generated due to symmetry breaking phase transitions in the early universe due to the Kibble mechanism [1]. The motion of these cosmic strings generate wakes behind them [2]. For specific value of energy, angular momentum and linear momentum, particles have closed orbits around the Abelian Higgs string. This leads to the trapping of the particles close to the string [3,4]. These strings once formed will move through the cosmic plasma generating wakes behind them. The plasma around the cosmic string consists of many particles. The energy of these particles depend on the plasma temperature at that time.

We are interested in studying neutrinos in the wakes of Abelian Higgs strings. Since neutrinos have mass, for certain values of angular momentum and energy, they will rotate in closed orbits around a static Abelian Higgs string. Due to the inherent lepton asymmetry in the universe, the rotating neutrinos will give rise to a neutrino current close to the string. Generally, cosmic strings are not stationary in the early universe. So the motion of the string will impart a velocity kick to the neutrinos. This velocity kick will enhance the density gradients generated by the neutrino current. As the string moves through the plasma, the neutrinos will interact with the background plasma. These non-uniform neutrino currents will generate inhomogeneities in the electron distribution in the background plasma [5].The inhomogeneous electron distribution will cause electric currents in the wake. The changing electric current will generate a magnetic field. We make an estimate of the magnitude of the magnetic field which can be generated in the wakes of these cosmic string.

Though bound electrons, which also have mass and will be rotating around the cosmic string generate a current, the positrons will also generate currents of equal magnitude but in opposite directions. So the net effective electromagnetic current is zero. However, in the case of neutrinos, due to the neutrino-antineutrino asymmetry in the early universe, a net neutrino current will be observed.

\section{Wakes due to cosmic strings}

Since the spacetime around a cosmic string is conical, a string moving with a velocity $v_{s}$ in a particular direction in a plane, will give a velocity perturbation $\Delta v$ to the surrounding particles. Since the velocity perturbation will be towards the string, it leads to an overdensity behind the cosmic string which is generally referred to as the cosmic string wake [2]. The string has a deficit angle given by $\delta \theta=8 \pi G \tilde{\mu}$, where $\tilde{\mu}$ is the mass per unit length of the string. As the string moves forward, matter will stream past it. The magnitude of the velocity perturbation imparted to the particles is given by $\delta v \sim \delta \theta v_{s} \gamma_{s}$, where $v_{s}$ is the velocity of the string and $\gamma_{s}$ is the relativistic factor. The strings generated in the early universe generally move at relativistic velocities. So a string moving at a time $t_{i}$ will generate a wake whose dimensions are given by $c_{1} t_{i} \times t_{i} v_{s} \gamma_{s} \times \delta \theta t_{i} v_{s} \gamma_{s}$. Here $c_{1}$ is a constant of order one. The opening angle of the wake depends upon the deficit angle of the cosmic string.

Generally, the plasma is charge neutral. So the overdensity too is charge neutral. As the string moves through the plasma, the velocity in the direction of motion is greater than the velocity in the other directions [6], this leads to the formation of shocks. Shocks formed at high temperatures will have a narrow opening angle and a high overdensity. Since the density of the plasma is related to 
the temperature, an overdensity in the shock will mean a temperature gradient in the plasma [7]. This temperature gradient $\nabla T$ can be calculated at a particular temperature of the plasma. For a general shock structure, the number density gradient and the temperature gradient are parallel to each other. However, this scenario will change for the case of neutrinos moving close to an Abelian Higgs string. This misalignment between the number density gradient and the temperature gradient will generate a magnetic field in the wake of the cosmic string.

\section{Neutrino current density around cosmic strings}

We have taken neutrinos as our massive particle. The Fermi distribution of these neutrinos in a rotating system is given by,

$$
f\left(E, l_{z}, \beta\right)=\left[\exp \left(\frac{E-l_{z} \Omega-\mu \beta}{T}\right)+1\right]^{-1}
$$

Here $\Omega$ is the angular velocity, $\mu$ is the chemical potential of the neutrinos, $l_{z}$ is the projection of the particle's total angular momentum on the direction of $\Omega$ and $E$ is the energy of the neutrinos. The factor $\beta$ takes on the values 1 or -1 depending on whether the neutrinos are more than the antineutrinos in the plasma. The neutrino field equations in the metric of the cosmic string.

$$
\gamma^{\mu}\left(\partial_{\mu}-\Gamma_{\mu}\right) \psi-m \psi=0
$$

Here $m$ denotes the mass of the neutrinos and $\Gamma_{\mu}$ is the spinor connections. On solving the equations using cylindrical line element and suitable Gamma matrices, the wavefunction is given by,

$$
\psi\left(E, p_{z}, l_{z}, \beta\right)=\frac{1}{4 \pi} e^{-i E t} e^{i p_{z} z} e^{-i l_{z} \phi}\left[\begin{array}{c}
\xi \\
\beta \xi
\end{array}\right]
$$

Where $p_{z}$ is the component of linear momentum in the $z$ direction and $J_{l_{z}+1 / 2}(\alpha r)$ are the Bessel functions [8]. The two-component spinor $\xi$ is given by,

$$
\xi=\left[\begin{array}{l}
i(E+m-p)^{1 / 2} J_{l_{z}+1 / 2}(\alpha r) \\
\beta(E+m+p)^{1 / 2} J_{l_{z}-1 / 2}(\alpha r)
\end{array}\right]
$$

Once the wavefunction is obtained, we can get the neutrino current. The total current density as a function of $r$ (the distance from the string axis) is given by[8],

$$
J(\alpha r)=\int_{0}^{\infty} d E \int_{-E}^{E} d p \sum_{\beta= \pm 1} \sum_{l_{z}} f\left(E, l_{z}, \beta\right) \beta \psi^{\dagger}\left(E, p_{z}, l_{z}, \beta\right) \gamma^{t} \gamma^{z} \psi\left(E, p_{z}, l_{z}, \beta\right)
$$

We obtain the neutrino current around the cosmic string by solving eqn.5 numerically. The cosmic string is generally characterized by it's symmetry breaking scale. The symmetry breaking scale is usually used to rescale the variables to make them dimensionless [3, 4]. We have chosen $E=1.083, l_{z}^{2}=0.025$ and $p_{z}=0.02$ to obtain the graph. The numerically obtained values of $J(\alpha r)$ are plotted in figure 1. The distance from the core is in terms of $\alpha r$. Here $\alpha=\sqrt{(E+m)^{2}-p_{z}^{2}}$. Hence $\alpha$ is in $G e V$ and we get the current in $G e V^{3}$. The neutrino current will also depend on the 
amount of lepton asymmetry in the plasma. But there is no significant change in the magnitude of the neutrino current in the range of $10^{-10}$ to $10^{-4}$.

The current is oscillatory in nature and decreases with distance from the string. The maximum value is close to the core of the cosmic string. Though the values are small, for the early universe plasma it is not negligible. These currents depend on the interaction cross section of the

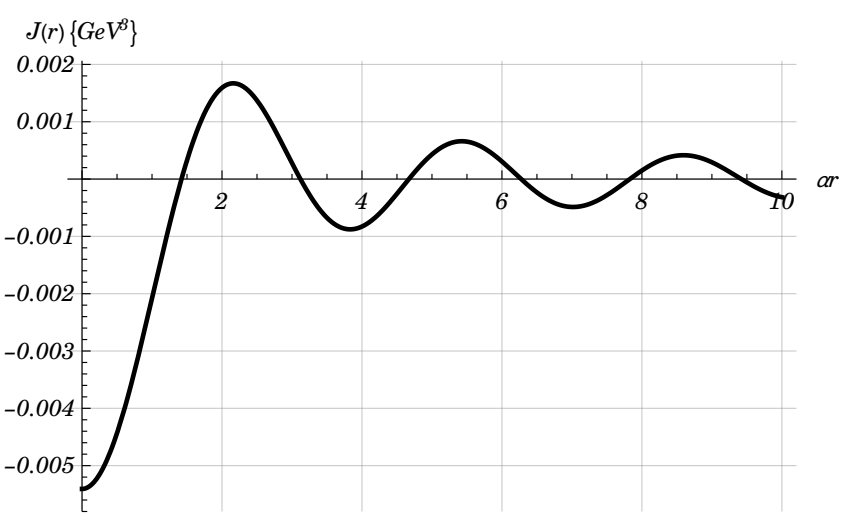

Figure 1: Neutrino current as a function of $r$ (distance from the cosmic string). Here $\alpha r$ is dimensionless. particles. This current is a directed flux of particles moving collectively through the plasma. We will use the estimate of the neutrino current to calculate the magnetic field. The oscillatory neutrino distribution will change with time due to the velocity of the moving string. The overdensity behind the string will also enhance the current further. The wake density is double the background density of the plasma. So the neutrino distribution will be less in front of the string while it will be much more behind the string.

\section{Neutrino currents in moving cosmic string wakes}

Neutrino interaction with the background plasma has been studied before [9]. Here neutrinoplasma interaction has been described by two-fluid model and neutrino currents generate an electromagnetic current in the early universe. We can obtain the continuity equations for these two-fluid plasma interaction [10]. We will use the perturbative approach to obtain the relation between the neutrino perturbation and the electron perturbation in the plasma. The two density perturbations are related by,

$$
\left(\frac{\partial^{2}}{\partial t^{2}}+\omega_{p}^{2}\right) \delta N_{e}=-\frac{\sqrt{2} G_{F} N_{e}}{m_{e} c^{2}}\left(\frac{\partial^{2}}{\partial t^{2}}-c^{2} \nabla^{2}\right) \delta N_{v}
$$

Here $\delta N_{e}$ is the electron number density fluctuation, $\delta N_{v}$ is the neutrino number density perturbation, $N_{e}$ is the mean electron density of the plasma and $\omega_{p}$ is the plasma oscillation frequency. The neutrino current in the plasma will thus generate a plasma potential $\phi_{e}$ due to the charge separation given by $\nabla^{2} \phi_{e}=4 \pi \delta N_{e}$. An electron current is thus generated in the shock wave.

Here there is no head on collision between the neutrino current and the string wake. For small scattering angle energy is transferred from the neutrinos to the plasma, however here the angles are closer to $\pi / 2$. This means that not much energy is transferred from the neutrinos to the plasma. So no instability is expected to be generated in the plasma. However, the Reynolds number in the plasma is very high. So we will have localized magnetic fields in the string's wake generated by the Biermann battery mechanism. In a two-fluid description of the plasma the magnetic field evolution, as given by the Biermann battery mechanism is [11],

$$
\frac{\partial \vec{B}_{e}}{\partial t}=\nabla \times\left(\vec{v}_{e} \times \vec{B}_{e}\right)+\frac{\eta_{r e s}}{4 \pi} \nabla^{2} \vec{B}_{e}-\frac{1}{e N_{e}} \nabla \times\left(\vec{j} \times \vec{B}_{e}\right)-\frac{1}{N_{e} e} \nabla N_{e} \times \nabla T_{e}
$$


Here, $\vec{v}_{e}$ is the electron fluid velocity, $N_{e}$ is the electron number density, $T_{e}$ is the electron temperature and $\eta_{r e s}$ is the resistivity of the plasma. The last term on the right hand side is the Biermann battery term. In the case where there is no magnetic field $\left(\vec{B}_{e}=0\right)$, this term generates the magnetic field due to the misalignment between the density and the temperature gradients of the electrons.

If the string is moving in the $y$-direction in the $x-y$ plane. Then the shock is stretched along the $y$ axis with a small width in the $x$-axis. The temperature gradient is primarily along the $x$-axis. Now the neutrino current exerts a force on the electrons, pushing them into the less neutrino abundant region, so the electron gradients will be complementary to the neutrino gradient caused by the Abelian Higgs strings. So, if the string was moving in the $y$-direction, they would also be in the $y$-direction.

Fig 1 plots the neutrino density with

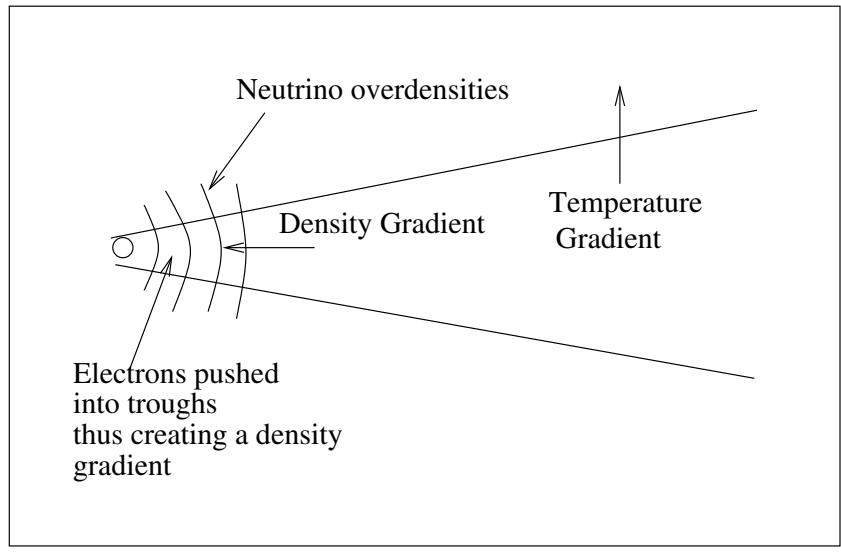

Figure 2: An illustration to show the generation of magnetic field in the wakes of cosmic strings due to the non uniform neutrino overdensities. respect to $\alpha r$, where $r$ is the radial distance away from the string, since $r=\sqrt{x^{2}+y^{2}}$, the neutrino gradient and the temperature gradient will not be parallel to each other. Since the neutrino gradient and the electron gradient will be complimentary to each other, therefore, the Biermann battery term will give rise to a finite magnetic field. In fig 2, we have given an illustration to explain the magnetic field generation. We make an order of magnitude estimate for fields generated around $100 \mathrm{GeV}$ temperature scales in the early universe. We assume that the density perturbation for neutrinos is proportional to the density perturbation of the electrons which is taken to be in the $y$ direction. The temperature gradient across a cosmic string shock (in the $x$-direction) is of the order of $10^{-5} T$ [12]. So from the Biermann mechanism,

$$
\frac{\partial B}{\partial t} \sim \frac{\sqrt{2} G_{F}}{m_{e} c^{2}} \frac{\partial N_{v}}{\partial y} \frac{\partial T_{e}}{\partial x}
$$

We consider $G_{F}=10^{-5} \mathrm{GeV}^{-2}, \frac{\partial N_{v}}{\partial y} \sim 0.02 \mathrm{GeV}^{3}$ and $\frac{\partial T_{e}}{\partial x} \sim 10^{-5} \times 200 \mathrm{GeV}$. The lengthscales at around the electroweak scales are about $1 \mathrm{GeV}^{-1}$ and if we take a similar time scale (about $1 \mathrm{GeV}^{-1}$ ) then the order of magnitude of the generated magnetic field is about $10^{13}$ Gauss. At the electroweak scale, the equipartition magnetic field is $\sim 10^{24} G$ [13]. So, this is not a very large field Though the field generated in the shock region is smaller than this, the Reynolds number is very high at such scale $\sim 10^{12}$, so it is quite possible that this small field can grow into a larger field due to turbulence.

\section{Summary and Conclusions}

Abelian Higgs strings moving through the plasma generate a neutrino current which has density gradients in the direction of motion of the string. The wake formed behind the string will have a cross perturbation across it. This cross-perturbation creates density discontinuities in the wake. 
These can be looked as interfaces through which the wakes pass. At high temperatures, shocks are formed in the wakes of cosmic strings. As the shocks cross the interface, the particles in the interface are accelerated by the shock wave. Due to the neutrino - electron interaction in the plasma, an electron current is generated in the plasma. The misaligned electron current and temperature gradient generate a magnetic field. We have obtained an order of magnitude estimate for the generated magnetic field and find that though low it is not negligible. Our estimate is very conservative as it does not include the effect of the high Reynolds numbers in the early universe. There are other mechanisms (such as bubble collisions at the electroweak scale ) which generate lower magnetic fields which are subsequently enhanced due to the high Reynolds number in the plasma. The detailed discussion on magnetic field generation by neutrino currents in cosmic string wakes may be obtained from ref. [14].

We would like to acknowledge discussions with Abhisek Saha and Prof. A. K. Kapoor.

\section{References}

[1] H. B. Nielsen and P. Olesen, Nucl. Phys. B 61 (1973) 45; M. Hindmarsh, S. Stuckey, and N. Bevis, Phys Rev D. 79. 123504, 2009 ;

[2] J. Silk and A. Vilenkin, Phys. Rev. Lett.53, 1700 (1984); M. J. Rees, Mon. Not. R. Astron. Soc. 222, 27 (1986); T. Vachaspati, Phys. Rev. Lett. 57, 1655 (1986);

[3] B. Hartmann, and P.J. Sirimachan, High Energ. Phys. (2010) 2010: 110.

[4] A. Saha and S. Sanyal, Journal of Cosmology and Astroparticle Physics 2018.03 (2018) 022.

[5] L. O Silva, R. Bingham, J. M . Dawson, W. B. Mori, Phys. Rev. E 592273 (1999).

[6] A. Stebbins, S. Veeraraghavan, R. H. Brandenberger, J. Silk, and N. Turok, Astrophys. J.322, 1 (1987).

[7] B. Layek, S. Sanyal and A. M. Srivastava, Physical Review D 63, 083512, (2001).

[8] A. Vilenkin, Physical Review D 20, 1807 (1979).

[9] L. O. Silva, R. Bingham, J. M. Dawson, J. T. Mendonça, and P. K. Shukla, Physics of Plasmas 7, $2166(2000)$

[10] A. Serbeto, Physics Letters A, 296, 217 (2002)

[11] K. M. Schoeffler, N. F. Loureiro, R. A. Fonseca, and L. O. Silva. Physics of Plasmas 23, no. 5 (2016): 056304

[12] B. Layek, Physical Review D. 71(6) 063527, (2005).

[13] G. Baym, D. Bodekar and L. McLerran, Physical Review D 53, 662 (1996).

[14] S. Sau, S. Sanyal, Eur. Phys. J. C 80, 152 (2020). 